\title{
A cost-utility analysis of apalutamide for metastatic castration-sensitive prostate cancer
}

Ambica Parmar ${ }^{1,2}$; Narhari Timilshina ${ }^{2,3}$; Urban Emmenegger ${ }^{1,4}$; Martin Smoragiewicz ${ }^{1}$; Beate Sander ${ }^{2,3,5,6} ;$ Shabbir Alibhai ${ }^{2,7} ;$ Kelvin K.W. Chan ${ }^{1,3,8}$

${ }^{1}$ Odette Cancer Centre, Sunnybrook Health Sciences Centre, Toronto, ON, Canada; ${ }^{2}$ Institute of Health Policy, Management and Evaluative Sciences, University of Toronto, Toronto, ON, Canada; ${ }^{3}$ Ontario Health, Toronto, ON, Canada; ${ }^{3}$ Toronto Health Economic and Technology Assessment Collaboration, University Health Network, Toronto, ON, Canada; ${ }^{4}$ Institute of Medical Science, University of Toronto, Toronto, ON, Canada; ${ }^{5}$ Public Health Ontario, Toronto, ON, Canada, ${ }^{6} \mathrm{ICES}$, Toronto, ON, Canada; ${ }^{7}$ Department of Medicine, Princess Margaret Cancer Centre, University Health Network, Toronto, ON, Canada; ${ }^{8}$ Canadian Applied Research in Cancer Control, Toronto, ON, Canada

Cite as: Parmar A, Timilshina N, Emmenegger U, et al. A cost-utility analysis of apalutamide for metastatic castration-sensitive prostate cancer. Can Urol Assoc J 2021 October 18; Epub ahead of print. http://dx.doi.org/10.5489/cuaj.7495

Published online October 18, 2021

Corresponding author: Dr. Kelvin K.W. Chan, Sunnybrook Health Sciences Centre, Toronto, ON, Canada; kelvin.chan@sunnybrook.ca

$* * *$

\section{Abstract}

Introduction: Earlier application of oral androgen receptor-axis-targeted therapies in patients with metastatic castration-sensitive prostate cancer (mCSPC) has established improvements in overall survival, as compared to androgen deprivation therapy (ADT) alone. Recently, the use of apalutamide plus ADT has demonstrated improvement in mCSPC-related mortality, vs. ADT alone, with an acceptable toxicity profile. However, the cost-effectiveness of this therapeutic option remains unknown.

Methods: We used a state-transition model with probabilistic analysis to compare apalutamide + ADT, as compared to ADT alone for mCSPC patients over a time horizon of 20 years. Primary outcomes included expected life-years (LY), quality-adjusted life-years (QALY), lifetime cost (2020 Canadian dollars), and incremental cost-effectiveness ratio (ICER). Parameter and model uncertainties were assessed through scenario analyses. Health outcomes and cost were discounted at $1.5 \%$, as per Canadian guidelines.

Results: For the base-case analysis, expected LY for ADT and apalutamide plus ADT were 4.11 and 5.56, respectively (incremental LY 1.45). Expected QALYs were 3.51 for ADT and 4.84 for 
apalutamide plus ADT (incremental QALYs 1.33); expected lifetime cost was $\$ 36582$ and $\$ 255$ 633 , respectively (incremental cost $\$ 219,051$ ). ICER for apalutamide plus ADT, as compared to ADT alone, was $\$ 164700 / \mathrm{QALY}$. Through scenario analysis, price reductions $\geq 50 \%$ were required for apalutamide in combination with ADT to be considered cost-effective, at a costeffectiveness threshold of $\$ 100$ 000/QALY.

Conclusions: Apalutamide plus ADT is unlikely to be cost-effective from the Canadian healthcare perspective unless there are substantial reductions in the price of apalutamide treatment.

\section{Introduction}

Since 2004, there has been a rapid expansion in life-prolonging systemic treatment options for patients with metastatic castration-resistant prostate cancer (mCRPC). ${ }^{1}$ More recently, these systemic treatments, including docetaxel, abiraterone and enzalutamide, have been evaluated earlier in the disease course, in patients with metastatic castration-sensitive prostate cancer (mCSPC) demonstrating significant improvements in both progression-free survival (PFS) and overall survival (OS) ${ }^{2-6}$ However, the toxicity profiles of these agents raise some concerns about the generalizability of these treatments. ${ }^{6-8}$

The androgen receptor-axis-targeted therapy (ARAT), apalutamide, is the fourth agent to demonstrate efficacy for patients with mCSPC. The phase III TITAN trial compared the addition of apalutamide to ADT versus ADT alone, demonstrating a significant improvement in both radiographic PFS [hazard ratio (HR): 0.48 (95\% confidence interval, CI: $0.39-0.60), \mathrm{p}<0.001$ ] and OS [HR: 0.65 (95\% CI): $0.53-0.79, \mathrm{p}<0.0001] .{ }^{9,10}$ This benefit was seen irrespective of volume of metastatic burden, in keeping with the volume-independent benefits demonstrated with abiraterone and enzalutamide. ${ }^{2,3,6}$ In addition, a tolerable toxicity profile was also demonstrated in the TITAN trial with grade $3 / 4$ adverse events (AE) occurring in $49 \%$ of patients, as compared to $42 \%$ in those treated with ADT alone. ${ }^{9,10}$

With improved efficacy and a tolerable toxicity profile, the use of apalutamide may be the preferred systemic therapy for patients with MCSPC. However monthly costs for apalutamide are estimated at upwards of $\$ 3,000$ per patient, necessitating the demonstration of costeffectiveness prior to recommendation. The objective of this study was to conduct a cost-utility analysis (CUA) of apalutamide in combination with ADT (apalutamide+ADT), in comparison to ADT alone, from the perspective of the publicly funded Canadian healthcare system. 


\section{Methods}

\section{Model overview}

A CUA using a state-transition model was used to compare the treatment strategies of apalutamide+ADT, versus ADT, as first-line systemic therapy for patients with mCSPC. The state-transition model consisted of three, mutually exclusive health states of progression-free $(\mathrm{PF})$, progressive-disease $(\mathrm{PD})$ and death.

The Canadian healthcare perspective was adopted for this analysis, incorporating only costs associated with publicly funded medical interventions. Primary outcomes included expected life-years, quality-adjusted life-years (QALY), lifetime cost and the incremental costeffectiveness ratio (ICER). Health outcomes and cost were calculated over an estimated lifetime horizon (i.e. 20 years) in one-month time steps (cycle length). As per Canadian guidelines, health outcomes and cost were discounted at $1.5 \%$ per annum. ${ }^{11}$

The model was implemented using TreeAge 2021 software (TreeAge Software Inc., Williamstown, MA, USA).

\section{Progression and survival estimates}

The published TITAN PFS and updated OS Kaplan-Meier curves were used to inform the transition probabilities between health states. The curves were digitized with Plot Digitizer software (http://plotdigitizer.sourceforge.net) to derive estimates of pseudo-individual patientlevel data, which were then used to generate fitted parametric survival curves (i.e. exponential, gamma, log-normal, log-logistic, Weibull). The best-fit parametric curve was derived according to best statistical fit (using the Akaike Information Criterion), visual-inspection and clinical plausibility. Based upon this the log-normal distribution was chosen to model the transitions from the PF to PD health states. The best-fit parametric curves were used to extrapolate survival beyond the trial duration to a lifetime time horizon. ${ }^{12,13}$ An exponential distribution was used to model the transition from the PD to death health state, based upon clinical plausibility and model calibration to observed data from the updated OS Kaplan-Meier survival curves from the TITAN trial. ${ }^{10}$ The statistical analysis for curve generation and fitting was completed using $\mathrm{R}$ software (R Core Team 2013. R: A language and environment for statistical computing. $\mathrm{R}$ foundation for Statistical Computing, Vienna, Austria).

Baseline mortality due to non-cancer related factors was also included in the transition from PF to death, as informed by Canadian-derived mortality tables for men aged 65 and older. 14

\section{Utility estimates}

Utility estimates for the PF and PD health state for both treatment strategies were informed by the published literature from United Kingdom (UK) derived estimates for patients with mCSPC for the PF health state and mCRPC for the PD health state. ${ }^{15-18}$ In order to capture a loss in 
health utility due to severe (i.e. grade 3/4) AE, disutilities for fall, fracture, rash and seizure, were also included, with base-values and duration informed by the published literature. ${ }^{19-22}$ (Table 1)

\section{Cost estimates}

Costs for apalutamide+ADT were derived from list price estimates, as per the pan-Canadian Oncology Drug Review (pCODR) recommendations for the use of apalutamide for nonmetastatic castration-resistant prostate cancer. ${ }^{23}$ Systemic therapy costs for post-progression treatment with abiraterone, bicalutamide, docetaxel chemotherapy and enzalutamide was incorporated into the respective PD health states, as per the TITAN trial, with cost estimates based upon list prices estimates and local institutional prices. ${ }^{10,23,24}$ Costs associated with physician visits and routine imaging during active systemic therapy were also included. (Supplemental Table 1)

Costs for $\mathrm{AE}$ of interest that are expected to result in hospital admission were included for grade $3 / 4$ fall, fracture and/or seizure, based upon rates informed by the TITAN trial. ${ }^{10}$ Costs were estimated from the Ontario Case Costing Initiative (OCCI) Cost Analysis Tool (CAT) as the mean (standard deviation) cost for hospitalization inclusive of direct patient costs only (i.e. nursing, diagnostic imaging, pharmacy and laboratory cost). ${ }^{25}$

A one-time cost for end-of-life care in hospital was incorporated into our model as a terminal cost for the PD health state. As the majority of patients receive their end-of-life care in hospital, this cost estimate was derived from the published literature based upon the reported mean length-of-stay in hospital and/or hospice at the end-of-life for Canadian patients with a diagnosis of cancer. ${ }^{26}$

All costs were inflated to 2020 Canadian dollars using the Canadian Consumer Price Index (www.bankofcanada.ca).

\section{Calibration and validation}

Model calibration was conducted to the published PFS and updated OS Kaplan-Meier survival curves from the TITAN trial for transition probability of PF to PD and transition probability of PD to death, respectively. (Supplemental Figure 1)

\section{Base-case analysis}

A probabilistic analysis was used to evaluate all primary outcomes for apalutamide+ADT as compared to ADT alone, for a base-case cohort of Canadian men with newly diagnosed mCSPC. Cost estimates were characterized by gamma distributions, as derived by the mean and standard error (SE). Health state utility estimates and probabilities for events were characterized by beta distributions, as derived from the mean and SE. (Supplemental Table 1) For estimates that did not have a value for SE, this was estimated as $25 \%$ of the expected range. Cholesky decomposition of the covariance matrix was used to correlate the parameters of the utilized lognormal distributions. The ICER was evaluated for apalutamide+ADT versus ADT alone. 


\section{Scenario analyses}

Scenario analyses were conducted to explore model uncertainty. This included two scenario analyses used to evaluate the uncertainty of the expected effectiveness estimates for the treatment strategies. First, a scenario analysis was completed with a within trial time horizon (i.e. 52 months) to evaluate the proportion of expected health outcomes and costs derived from observable data, as compared to long-term expected outcomes. In addition, the uncertainty in the post-progression effectiveness estimates for apalutamide+ADT was evaluated through a scenario analysis of alternative expected mortality rates, as modeled with alternative rate parameters in the exponential distribution for the transition from the PD health state to death, to estimate varying 5-year survival expectations.

Additionally, scenario analyses with alternative probabilities of post-progression systemic treatment following either treatment strategies were conducted, given the uncertainty in these estimates due to short follow-up in the TITAN trial.

A scenario analysis of price reductions for apalutamide was also conducted, given the high cost of treatment with apalutamide as compared to ADT, by evaluating the expected costs and ICER through price reduction of apalutamide by $25 \%, 50 \%$ and $75 \%$.

\section{Results}

\section{Base case analysis}

Apalutamide+ADT was associated with 5.56 expected life-years, as compared to 4.11 with ADT. QALYs for apalutamide+ADT and ADT were 4.84 and 3.51, respectively. The expected lifetime cost of apalutamide+ADT and ADT were $\$ 255,633$ and $\$ 36,582$, respectively. The resultant ICER for apalutamide+ADT versus ADT was $\$ 164,700 / \mathrm{QALY}$. Table 1 presents the disaggregate health outcomes and cost for each strategy.

Figure 1 depicts the cost-effectiveness acceptability curve. At cost-effectiveness thresholds less than $\$ 100,000 /$ QALY, ADT was the preferred therapeutic strategy.

\section{Scenario analyses}

In the scenario analysis conducted using a within trial time horizon from the updated OS analysis, apalutamide+ADT and ADT generated QALYs of 2.61 and 2.29, respectively, with an incremental gain in QALY of 0.32 with the combination. The incremental cost was $\$ 114,487$ with the addition of apalutamide to ADT, with a resultant ICER of \$357,772/QALY. (Table 2) The incremental QALY within trial time horizon made up 24\% of the expected lifetime incremental QALYs while the incremental cost within trial time horizon made up 52\% of expected lifetime incremental cost, for apalutamide+ADT versus ADT.

The scenario analysis with different expected mortality rates post-progression with apalutamide+ADT is summarized in Supplemental Figure 2. With an expected 5-year survival rate of $67 \%$ the ICER improved to $\$ 104,904 /$ QALY. 
Given the short trial follow-up a scenario analysis of alternative probabilities of subsequent therapy was conducted, as summarized in Table 3. Although higher probabilities of subsequent therapy led to higher costs, no substantial difference in the resultant ICER was seen. Similarly, the ICER remained $>\$ 160,000 /$ QALY in the scenario where higher rates of subsequent therapy were only applied following initial treatment with ADT alone.

In the scenario analysis by price reduction for apalutamide, at a price reduction of $50 \%$, apalutamide+ADT demonstrated an ICER of $\$ 87,567 /$ QALY. (Supplemental Table 2)

\section{Discussion}

Apalutamide+ADT resulted in an improvement in health outcomes as demonstrated by an improvement in both life-years and QALYs, as compared to ADT alone. However, the combination treatment also generated higher expected lifetime costs. With a resultant ICER of $\$ 164,700 /$ QALY, apalutamide+ADT was not found to be cost-effective from the perspective of the Canadian, public-payer, healthcare system at current list prices. However, apalutamide+ADT may be cost-effective at a cost-effectiveness threshold of $\$ 100,000 /$ QALY with a price reduction of $50 \%$ or more.

Our analysis supports the clinical effectiveness of apalutamide+ADT versus ADT alone, as evidenced by improvements in both life-years and QALYs. Consistent with clinical trial efficacy data, the clinical benefit for apalutamide+ADT was mostly driven by gains in health outcomes in the mCSPC setting. ${ }^{9}$ However, with a longer time spent in the mCSPC health state, patients treated with apalutamide+ADT also accrued higher expected costs in this health state, as compared to ADT. Accordingly, the lack of cost-effectiveness of apalutamide+ADT is largely driven by the high recurrent drug cost of apalutamide. As such, to improve cost-effectiveness, it is evident that reductions in the price of apalutamide are required, a conclusion supported by our scenario analyses.

The result of our scenario analysis within trial time horizon highlights the need for caution when interpreting expected, as compared to observed, data on health outcomes and cost. For both treatment strategies, the expected gains in QALYs and costs within trial time horizon represented less than $60 \%$ of the expected outcomes for apalutamide+ADT and less than $70 \%$ of the expected outcomes of ADT over a lifetime horizon. This highlights the impact of extrapolated outcomes on total expected lifetime estimates. As such, this highlights the need for re-evaluation with ongoing maturity of the TITAN trial, to more accurately assess the costeffectiveness of this novel combination.

Across abiraterone, apalutamide and enzalutamide, there is no clear superior choice for systemic therapy in mCSPC with similar demonstrated efficacy, through improvement in PFS and/or OS, seen with all three agents. ${ }^{2,3,6,9,15,27}$ Although these therapies have the potential to lead to significant health benefits, their substantial costs highlight the need for demonstration of cost-effectiveness prior to adoption. Prior evaluation of the cost-effectiveness of abiraterone, in combination with ADT as compared to ADT alone, and as compared to docetaxel have been 
completed. ${ }^{18,28-30}$ Across these cost-effectiveness analyses of abiraterone plus ADT, none have demonstrated cost-effectiveness for abiraterone at current prices. A recently published costeffectiveness analyses comparing all three ARATs, docetaxel plus ADT and ADT alone found abiraterone plus ADT to be the most cost-effective systemic therapy for mCSPC from the US payer perspective. ${ }^{31}$ Future cost-effectiveness analyses of all available systemic therapy agents in mCSPC from the Canadian healthcare perspective is warranted.

Notable limitations of our analysis include the absence of granular data to inform all cost and effectiveness estimates. For instance, this model included only one line of therapy for patients who transitioned into the MCRPC setting which may have misrepresented the included costs with both treatment strategies. There may also be underrepresentation of total healthcare costs, as only costs associated with select grade $3 / 4 \mathrm{AE}$ were included. Further, in the absence of published results for Canadian-specific preference-based estimates for quality-of-life in the PF and PD health state, utilities from the published literature based upon UK populations were utilized, which may not be entirely representative of the Canadian population. ${ }^{32}$

\section{Conclusions}

Apalutamide in combination with ADT as first-line systemic therapy in the management of mCSPC was not found to be cost-effective at the current list price from the Canadian healthcare perspective. Cost-effectiveness may be improved with price reductions of apalutamide. Reevaluation of cost-effectiveness with ongoing maturity of survival data is warranted to reduce the uncertainty in cost-effectiveness conclusions. 


\section{References}

1. Sartor O, de Bono JS: Metastatic Prostate Cancer. N Engl J Med 378:645-657, 2018

2. James ND, de Bono JS, Spears MR, et al: Abiraterone for Prostate Cancer Not Previously Treated with Hormone Therapy. N Engl J Med 377:338-351, 2017

3. Fizazi K, Chi KN: Abiraterone in Metastatic Prostate Cancer. N Engl J Med 377:1697-8, 2017

4. Gravis G, Fizazi K, Joly F, et al: Androgen-deprivation therapy alone or with docetaxel in non-castrate metastatic prostate cancer (GETUG-AFU 15): a randomised, open-label, phase 3 trial. Lancet Oncol 14:149-58, 2013

5. Sweeney CJ, Chen YH, Carducci M, et al: Chemohormonal Therapy in Metastatic Hormone-Sensitive Prostate Cancer. N Engl J Med 373:737-46, 2015

6. Davis ID, Martin AJ, Stockler MR, et al: Enzalutamide with Standard First-Line Therapy in Metastatic Prostate Cancer. N Engl J Med 381:121-131, 2019

7. Lavoie JM, Zou K, Khalaf D, et al: Clinical effectiveness of docetaxel for castrationsensitive prostate cancer in a real-world population-based analysis. Prostate 79:281-287, 2019

8. Moreira RB, Debiasi M, Francini E, et al: Differential side effects profile in patients with mCRPC treated with abiraterone or enzalutamide: a meta-analysis of randomized controlled trials. Oncotarget 8:84572-84578, 2017

9. Chi KN, Agarwal N, Bjartell A, et al: Apalutamide for Metastatic, Castration-Sensitive Prostate Cancer. N Engl J Med 381:13-24, 2019

10. Chi KN, Chowdhury S, Bjartell A, et al: Apalutamide in Patients With Metastatic Castration-Sensitive Prostate Cancer: Final Survival Analysis of the Randomized, Double-Blind, Phase III TITAN Study. J Clin Oncol 39:2294-2303, 2021

11. Canadian Agency for Drugs and Technology in Health: Guidelines for the economic evaluation of health technologies: Canada. Ottawa, 2017.

12. Guyot P, Ades AE, Ouwens MJ, et al: Enhanced secondary analysis of survival data: reconstructing the data from published Kaplan-Meier survival curves. BMC Med Res Methodol 12:9, 2012

13. Saluja R, Cheng S, Delos Santos KA, et al: Estimating hazard ratios from published Kaplan-Meier survival curves: A methods validation study. Res Synth Methods 10:465475, 2019

14. Statistics Canada: Life Tables, Canada, Provinces and Territories, 2019. Retrieved from https://www150.statcan.gc.ca/n1/en/catalogue/84-537-X

15. Chi KN, Protheroe A, Rodriguez-Antolin A, et al: Patient-reported outcomes following abiraterone acetate plus prednisone added to androgen deprivation therapy in patients with newly diagnosed metastatic castration-naive prostate cancer (LATITUDE): an international, randomised phase 3 trial. Lancet Oncol 19:194-206, 2018

16. Hall F, de Freitas HM, Kerr C, et al: Estimating utilities/disutilities for high-risk metastatic hormone-sensitive prostate cancer (mHSPC) and treatment-related adverse events. Qual Life Res 28:1191-1199, 2019

17. Lloyd AJ, Kerr C, Penton J, et al: Health-Related Quality of Life and Health Utilities in Metastatic Castrate-Resistant Prostate Cancer: A Survey Capturing Experiences from a Diverse Sample of UK Patients. Value Health 18:1152-7, 2015 
18. Sathianathen NJ, Alarid-Escudero F, Kuntz KM, et al: A Cost-effectiveness Analysis of Systemic Therapy for Metastatic Hormone-sensitive Prostate Cancer. Eur Urol Oncol 2:649-655, 2019

19. Doyle S, Lloyd A, Walker M: Health state utility scores in advanced non-small cell lung cancer. Lung Cancer 62:374-80, 2008

20. Matza LS, Chung K, Van Brunt K, et al: Health state utilities for skeletal-related events secondary to bone metastases. Eur J Health Econ 15:7-18, 2014

21. Nafees B, Stafford M, Gavriel S, et al: Health state utilities for non small cell lung cancer. Health Qual Life Outcomes 6:84, 2008

22. The National Institute for Health and Care Excellence: The Epilepsies: Clinical Practice Guidelines, 2010. Retrieved from https://www.nice.org.uk/guidance/cg137/documents/epilepsy-update-full-guidelineappendix-p2

23. Canadian Agency for Drugs and Technology in Health: Erleada for Castrate Resistant Prostate Cancer, 2018. Retrieved from https://www.cadth.ca/erleada-castrate-resistantprostate-cancer-details

24. Canadian Agency for Drugs and Technology in Health: Zytiga for metastatic castration resistant prostate cancer, 2013. Retrieved from https://www.cadth.ca/zytiga-metastaticcastration-resistant-prostate-cancer-details

25. Ministry of Health and Long Term Care: Ontario Case Costing Initiative, 2019. Retrieved from https://hsim.health.gov.on.ca/hdbportal/?destination=front page

26. Bekelman JE, Halpern SD, Blankart CR, et al: Comparison of Site of Death, Health Care Utilization, and Hospital Expenditures for Patients Dying With Cancer in 7 Developed Countries. JAMA 315:272-83, 2016

27. Agarwal N, McQuarrie K, Bjartell A, et al: Health-related quality of life after apalutamide treatment in patients with metastatic castration-sensitive prostate cancer (TITAN): a randomised, placebo-controlled, phase 3 study. Lancet Oncol 20:1518-1530, 2019

28. Aguiar PN, Jr., Tan PS, Simko S, et al: Cost-effectiveness analysis of abiraterone, docetaxel or placebo plus androgen deprivation therapy for hormone-sensitive advanced prostate cancer. Einstein (Sao Paulo) 17:eGS4414, 2019

29. Chiang CL, So TH, Lam TC, et al: Cost-effectiveness analysis of Abiraterone Acetate versus Docetaxel in the management of metastatic castration-sensitive prostate cancer: Hong Kong's perspective. Prostate Cancer Prostatic Dis, 2019

30. Hird AE, Magee DE, Cheung DC, et al: Abiraterone vs. docetaxel for metastatic hormone-sensitive prostate cancer: A microsimulation model. Can Urol Assoc J 14:E418E427, 2020

31. Sung WWY, Choi HCW, Luk PHY, et al: A Cost-Effectiveness Analysis of Systemic Therapy for Metastatic Hormone-Sensitive Prostate Cancer. Front Oncol 11:627083, 2021

32. Heijink R, Reitmeir P, Leidl R: International comparison of experience-based health state values at the population level. Health Qual Life Outcomes 15:138, 2017 


\section{Figures and Tables}

Fig. 1. Cost-effectiveness acceptability curve. Cost-effectiveness acceptability curve demonstrating the cost-effective strategy over a range of cost-effectiveness thresholds. Abbreviations: ADT: androgen deprivation therapy; QALY: quality-adjusted life-year.

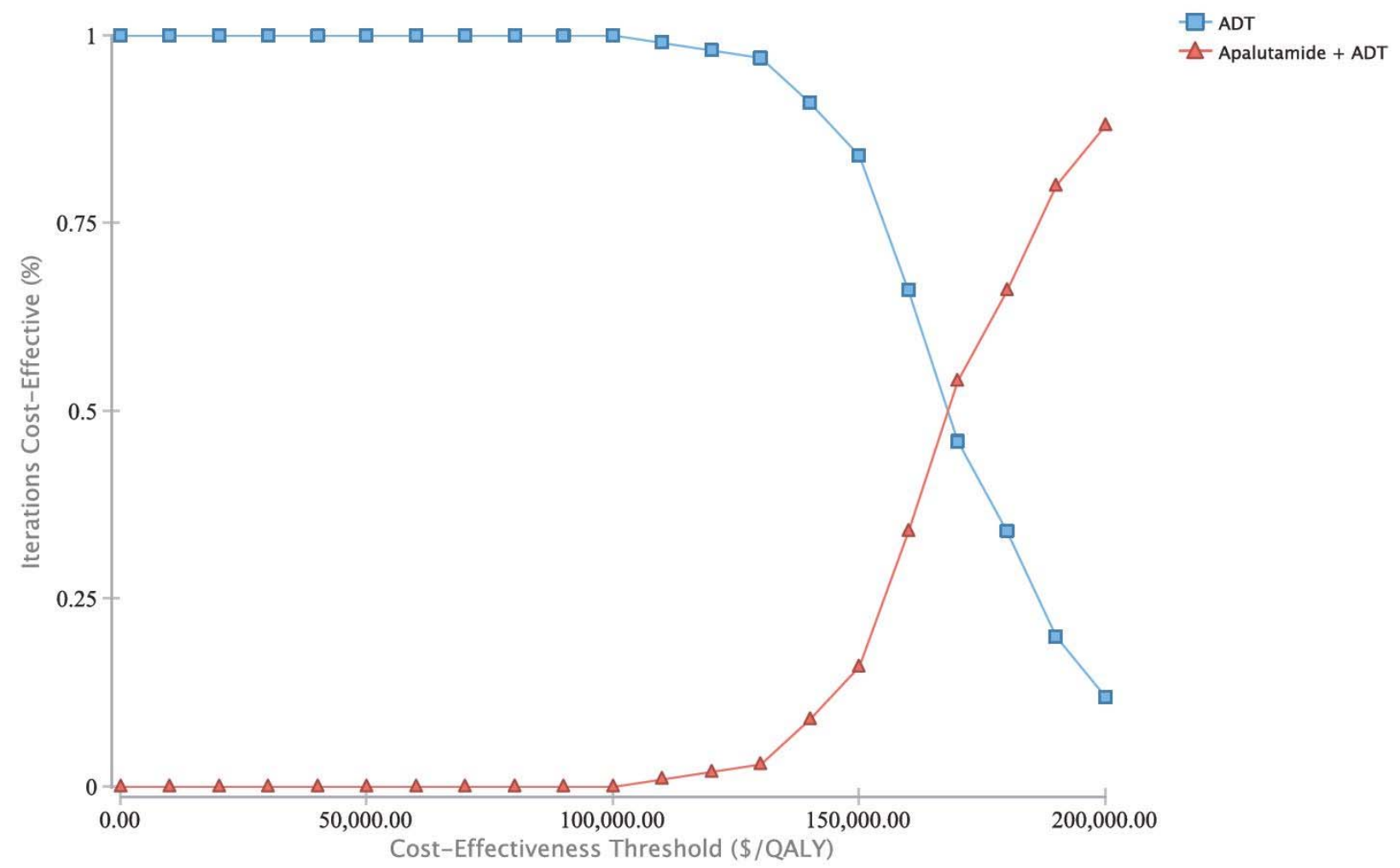




\begin{tabular}{|l|c|c|c|c|}
\hline Table 1. Base-case analysis \\
\hline Treatment strategy & $\begin{array}{c}\text { Life- } \\
\text { years }\end{array}$ & QALY & Cost (\$) & \\
\hline Apalutamide + ADT & 5.56 & 4.84 & 255633 & - \\
Progression-free & 4.71 & 4.19 & 240021 & \\
Post-progression & 0.85 & 0.65 & 15612 & - \\
\hline ADT & 4.11 & 3.51 & 36582 & - \\
Progression-free & 2.89 & 2.57 & 18549 & \\
Post-progression & 1.22 & 0.94 & 18033 & \$142 767/LYG \\
\hline Difference & 1.45 & 1.33 & 219051 & $\$ 151070 / \mathrm{LYG}$ \\
\hline Incremental cost-effectiveness ratio [cost (\$)/LYG] & $\$ 157$ 996/QALY \\
Undiscounted & & $\$ 164700 / \mathrm{QALY}$ \\
\hline
\end{tabular}

Disaggregated health outcomes (life-years and QALYs) and costs for treatment with ADT with and without apalutamide. Incremental cost-effectiveness ratio, per LYG and QALY, for treatment with apalutamide + ADT, as compared to ADT alone demonstrated. All costs represented in 2018 Canadian dollars. ADT: androgen-deprivation therapy; LYG: life-year gain; QALY: quality-adjusted life-year.

\begin{tabular}{|c|c|c|c|c|c|c|}
\hline \multirow{2}{*}{ Treatment strategy } & \multicolumn{3}{|c|}{ QALY } & \multicolumn{3}{|c|}{ Cost (\$) } \\
\hline & Lifetime & Trial & $\%$ & Lifetime & Trial & $\%$ \\
\hline Apalutamide + ADT & 4.84 & 2.61 & 54 & 255633 & 138566 & 54 \\
\hline Progression-free & 4.19 & 2.29 & 55 & 240021 & 130986 & 55 \\
\hline Post-progression & 0.65 & 0.32 & 49 & 15612 & 7580 & 49 \\
\hline ADT & 3.51 & 2.29 & 65 & 36582 & 24079 & 66 \\
\hline Progression-free & 2.57 & 1.72 & 67 & 18549 & 12394 & 67 \\
\hline Post-progression & 0.94 & 0.57 & 61 & 18033 & 11685 & 65 \\
\hline Difference & 1.33 & 0.32 & 24 & 219051 & 114487 & 52 \\
\hline \multicolumn{5}{|c|}{ Incremental cost-effectiveness ratio [cost (\$)/QALY] } & \multicolumn{2}{|c|}{ \$357 772/QALY } \\
\hline
\end{tabular}

Disaggregated health outcomes and cost for treatment with apalutamide with and without ADT using a within trial time-horizon (i.e., 52 months), represented as the proportion of expected health outcomes and cost in a lifetime time-horizon. ADT: androgen-deprivation therapy; QALY: quality-adjusted life-year. 


\begin{tabular}{|c|c|c|c|c|}
\hline Scenario & Treatment strategy & QALY & Cost (\$) & ICER \\
\hline \multirow{3}{*}{$\begin{array}{l}\text { Equal probabilities } \\
\text { to base-case } \\
\text { following ADT }\end{array}$} & Apalutamide + ADT & 4.84 & 255578 & - \\
\hline & ADT & 3.51 & 36582 & - \\
\hline & Incremental & 1.33 & 218996 & \$164 659/QALY \\
\hline \multirow{3}{*}{$\begin{array}{l}\text { Two-times the } \\
\text { base-case } \\
\text { probability for } \\
\text { subsequent therapy } \\
\text { following ADT } \\
\text { (equal) }\end{array}$} & Apalutamide + ADT & 4.84 & 256283 & - \\
\hline & ADT & 3.51 & 38221 & - \\
\hline & Incremental & 1.33 & 218062 & \$163 956/QALY \\
\hline \multirow{3}{*}{$\begin{array}{l}\text { Two-times the } \\
\text { base-case } \\
\text { probability for } \\
\text { subsequent therapy } \\
\text { following ADT } \\
\text { (for ADT only) }\end{array}$} & Apalutamide + ADT & 4.84 & 253633 & - \\
\hline & ADT & 3.51 & 38221 & - \\
\hline & Incremental & 1.33 & 215412 & \$161 964/QALY \\
\hline \multirow{3}{*}{$\begin{array}{l}\text { Three-times the } \\
\text { base-case } \\
\text { probability for } \\
\text { subsequent therapy } \\
\text { following ADT } \\
\text { (equal) }\end{array}$} & Apalutamide + ADT & 4.84 & 257,823 & - \\
\hline & ADT & 3.51 & 39553 & - \\
\hline & Incremental & 1.33 & 218270 & \$164 113/QALY \\
\hline \multirow{3}{*}{$\begin{array}{l}\text { Three-times the } \\
\text { base-case } \\
\text { probability for } \\
\text { subsequent therapy } \\
\text { following ADT } \\
\text { (for ADT only) }\end{array}$} & Apalutamide + ADT & 4.84 & 253633 & - \\
\hline & $\mathrm{AD}$ & 3.51 & 39553 & - \\
\hline & Incremental & 1.33 & 214080 & \$160 962/QALY \\
\hline
\end{tabular}

Scenario analysis of different rates of post-progression therapy, including: a) subsequent therapy for apalutamide+ADT equal to rates reported following ADT from TITAN trial; b) subsequent therapy estimated as twice the reported rate following ADT (applied to both strategies); c) subsequent therapy estimated as twice the reported rate following ADT (applied to the ADT strategy only); d) subsequent therapy estimated as three-times the reported rate following ADT (applied to both strategies); e) subsequent therapy estimated as three-times the reporting rate following ADT (applied to the ADT strategy only). ADT: androgen-deprivation therapy; ICER: incremental cost-effectiveness ratio; QALY: quality-adjusted life-year. 